Research Article

\title{
Degenerate Analogues of Euler Zeta, Digamma, and Polygamma Functions
}

\author{
Fuli He $\mathbb{D}^{1},{ }^{1}$ Ahmed Bakhet $\mathbb{D}^{1,2}{ }^{1,2}$ Mohamed Akel, ${ }^{3}$ and Mohamed Abdalla $\mathbb{D}^{3,4}$ \\ ${ }^{1}$ School of Mathematics and Statistics, Central South University, Changsha 410083, China \\ ${ }^{2}$ Department of Mathematics, Faculty of Science, Al-Azhar University, Assiut 71524, Egypt \\ ${ }^{3}$ Department of Mathematics, Faculty of Science, South Valley University, Qena 83523, Egypt \\ ${ }^{4}$ Department of Mathematics, College of Science, King Khalid University, Abha 61471, Saudi Arabia \\ Correspondence should be addressed to Mohamed Abdalla; moabdalla@kku.edu.sa
}

Received 23 February 2020; Accepted 7 April 2020; Published 6 May 2020

Guest Editor: Praveen Agarwal

Copyright (c 2020 Fuli He et al. This is an open access article distributed under the Creative Commons Attribution License, which permits unrestricted use, distribution, and reproduction in any medium, provided the original work is properly cited.

\begin{abstract}
In recent years, much attention has been paid to the role of degenerate versions of special functions and polynomials in mathematical physics and engineering. In the present paper, we introduce a degenerate Euler zeta function, a degenerate digamma function, and a degenerate polygamma function. We present several properties, recurrence relations, infinite series, and integral representations for these functions. Furthermore, we establish identities involving hypergeometric functions in terms of degenerate digamma function.
\end{abstract}

\section{Introduction}

The gamma, digamma, and polygamma functions have an increasing and recognized role in fractional differential equations, mathematical physics, the theory of special functions, statistics, probability theory, and the theory of infinite series. The reader may refer, for example, to [1-9]. These functions are directly connected with a variety of special functions such as zeta function, Clausen's function, and hypergeometric functions. The evaluations of series involving Riemann zeta function $\zeta(s)$ and related functions have a long history that can be traced back to Christian Goldbach (1690-1764) and Leonhard Euler (1707-1783) (see, for details, [10]). The Euler zeta function and its generalizations and extensions have been widely studied [11-15].

Later on, these functions arise in the study of matrixvalued special functions and in the theory of matrix-valued orthogonal polynomials, see e.g., [16-23] and the references therein.

Motivated by this great importance of these functions, their investigations and generalizations to the degenerate framework have been widely considered in the literature, for instance, [24-27].

In this section, we present some basic properties and well-known results on a degenerate gamma function which we need in this work. In Section 2, we introduce a degenerate Euler zeta function and discuss its region of convergence, integral representation, and infinite series representation. In Section 3, we define a degenerate digamma function along with its region of convergence and integral representation. We also give certain recurrence relations and formulae satisfied by the degenerate digamma function. In Section 4, we define a degenerate polygamma function and describe its convergence conditions. Some recurrence relations satisfied by the degenerate polygamma function are also given here. Finally, in Section 5, the hypergeometric functions are expressed in terms of the degenerate digamma function.

In [26], a degenerate gamma function, denoted $\Gamma_{\lambda}^{*}$, has been defined by

$$
\Gamma_{\lambda}^{*}(z)=\int_{0}^{\infty}(1+\lambda)^{-t / \lambda} t^{z-1} \mathrm{~d} t, \quad \lambda \in(0,1), \operatorname{Re}(z)>0 .
$$


The basic results of this function, given in [26], can be summarized in the following lemma.

Lemma 1. Let $\lambda \in(0,1)$. Then, for $z \in \mathbb{C}$ with $\operatorname{Re}(z)>0, \Gamma_{\lambda}^{*}(z)$ satisfies

$$
\begin{aligned}
\Gamma_{\lambda}^{*}(z+1) & =\frac{\lambda z}{\log (1+\lambda)} \Gamma_{\lambda}^{*}(z) \\
\Gamma_{\lambda}^{*}(1) & =\frac{\lambda}{\log (1+\lambda)}, \\
\Gamma_{\lambda}^{*}(z+1) & =\frac{\lambda^{k+1} z(z-1) \cdots(z-k)}{(\log (1+\lambda))^{k+1}} \Gamma_{\lambda}^{*}(z-k), \quad k \geq 0 \\
\Gamma_{\lambda}^{*}(k+1) & =\frac{\lambda^{k+1} k !}{(\log (1+\lambda))^{k+1}}, \quad k \in \mathbb{N} .
\end{aligned}
$$

Also, we can easily show that

Corollary 1. Let $\lambda \in(0,1)$. Then, $\Gamma_{\lambda}^{*}(z)$ satisfies

$$
\Gamma_{\lambda}^{*}(z)=\left[\frac{\lambda}{\log (1+\lambda)}\right]^{z} \Gamma(z), \quad z \in \mathbb{C}, \operatorname{Re}(z)>0,
$$

where $\Gamma(z)$ is the gamma function. Moreover, for $m, n \in \mathcal{N}$, we have

$$
\Gamma_{\lambda}^{*}(m) \Gamma_{\lambda}^{*}(n)=B(m, n) \Gamma_{\lambda}^{*}(m+n),
$$

where $B(.,$.$) is the beta function.$

\section{Degenerate Euler Zeta Function}

The Euler zeta function in two complex variables $s, z$ such that $\operatorname{Re}(s)>0$ and $\operatorname{Re}(z)>0$ is defined by (see $[12,24])$

$$
\zeta_{E}(s, z)=2 \sum_{n=0}^{\infty} \frac{(-1)^{n}}{(n+z)^{s}} .
$$

An integral representation of $\zeta_{E}(s, z)$ is given as

$$
\zeta_{E}(s, z)=\Gamma^{-1}(s) \int_{0}^{\infty} F(-t, z) t^{s-1} \mathrm{~d} t,
$$

where

$$
F(t, z)=\frac{2 e^{z t}}{1+e^{t}}=\sum_{n=0}^{\infty} E_{n}(z) \frac{t^{n}}{n !}
$$

where $E_{n}(z)$ is the Euler polynomial of degree $n$. When $z=0, E_{n}=E_{n}(0)$ are Euler numbers (see, $\left.[12,14]\right)$. Kim in [14] obtained that $\zeta_{n}(-n, z)=E_{n}(z), n \geq 0$.

In this section, we consider a degenerate analogue of the Euler zeta function which is given as

$$
\zeta_{E_{\lambda}}(s, z)=\Gamma^{-1}(s) \int_{0}^{\infty} F_{\lambda}(-t, z) t^{s-1} \mathrm{~d} t
$$

where $\lambda \in(0,1), s, z \in \mathbb{C}$ with $\operatorname{Re}(s)>0, \operatorname{Re}(z)>0$, and

$$
F_{\lambda}(t, z)=\frac{2}{1+(1+\lambda)^{t / \lambda}}(1+\lambda)^{z t / \lambda}=\sum_{n=0}^{\infty} \mathscr{E}_{n}^{\lambda}(z) \frac{t^{n}}{n !} .
$$

By (9) and (11), it follows that

$$
\mathscr{E}_{n}^{\lambda}(z)=\left(\frac{\lambda}{\ln (1+\lambda)}\right)^{n} E_{n}(z)
$$

which is the degenerate Euler polynomial of degree $n$.

From (10) and (11), we obtain that

$$
\begin{aligned}
\Gamma^{-1}(s) \int_{0}^{\infty} F_{\lambda}(-t, z) t^{s-1} \mathrm{~d} t & =\Gamma^{-1}(s) \int_{0}^{\infty} 2 \sum_{m=0}^{\infty}(-1)^{m}(1+\lambda)^{-(m+z) t / \lambda} t^{s-1} \mathrm{~d} t \\
& =2 \Gamma^{-1}(s) \sum_{m=0}^{\infty}(-1)^{m} \int_{0}^{\infty}(1+\lambda)^{-\tau / \lambda} \frac{\tau^{s-1}}{(m+z)^{s}} \mathrm{~d} \tau \\
& =2 \frac{\Gamma_{\lambda}^{*}(s)}{\Gamma(s)} \sum_{m=0}^{\infty}(-1)^{m} \frac{1}{(m+z)^{s}} .
\end{aligned}
$$
result.

Thus, using (10) and (13), we conclude the following

Theorem 1. For $s, z \in \mathbb{C}$ with $\operatorname{Re}(s)>0, \operatorname{Re}(z)>0$, and $\lambda \in(0,1)$, the degenerate Euler zeta function $\zeta_{E_{\lambda}}(s, z)$ defined in (10) has the following infinite series representation:

$$
\zeta_{E_{\lambda}}(s, z)=2 \frac{\Gamma_{\lambda}^{*}(s)}{\Gamma(s)} \sum_{m=0}^{\infty}(-1)^{m} \frac{1}{(m+z)^{s}} .
$$

Moreover, in view of (5), we have

$$
\zeta_{E_{\lambda}}(s, z)=\zeta_{E}(s, z)\left(\frac{\lambda}{\ln (1+\lambda)}\right)^{s}
$$

where $\zeta_{E}(s, z)$ is the Euler zeta function defined by (7).

Furthermore, from (11), it follows

$$
\Gamma^{-1}(s) \int_{0}^{\infty} F_{\lambda}(-t, z) t^{s-1} \mathrm{~d} t=\Gamma^{-1}(s) \sum_{m=0}^{\infty} \mathscr{E}_{n}^{\lambda}(z) \frac{(-1)^{m}}{m !} \int_{0}^{\infty} t^{s+m-1} \mathrm{~d} t .
$$

Hence, we obtain the following results. 
Theorem 2. For $s, z \in \mathbb{C}$ with $\operatorname{Re}(s)>0, \operatorname{Re}(z)>0$, and $\lambda \in(0,1)$, the degenerate Euler zeta function $\zeta_{E_{\lambda}}(s, z)$, defined in (10), satisfies

$$
\zeta_{E_{\lambda}}(s, z)=\Gamma^{-1}(s) \sum_{m=0}^{\infty} \mathscr{E}_{n}^{\lambda}(z) \frac{(-1)^{m}}{m !} \int_{0}^{\infty} t^{s+m-1} \mathrm{~d} t .
$$

And for $n \in \mathbb{N} \cup\{0\}$,

$$
\zeta_{E_{\lambda}}(-n, z)=\frac{2 \pi i(-1)^{n}}{n ! \Gamma(-n)} \mathscr{E}_{n}^{\lambda}=\mathscr{E}_{n}^{\lambda}(z)
$$

Remark 1. Note that $\zeta_{E_{\lambda}}(s, z)$ is an entire function in the complex $s$-plane.

Remark 2.

$$
\lim _{\lambda \longrightarrow 0} \zeta_{E_{\lambda}}(-n, z)=E_{n}(z)=\zeta_{E}(-n, z) .
$$

\section{Degenerate Digamma Function}

The digamma function, denoted by $\psi(z)$, is the logarithmic derivative of the gamma function given by $[6,16,28]$ :

$$
\psi(z)=\frac{\mathrm{d}}{\mathrm{d} z} \log \Gamma(z)=\frac{\Gamma^{\prime}(z)}{\Gamma(z)} .
$$

In this section, we define a degenerate digamma function as follows:

$$
\psi_{\lambda}^{*}(z)=\frac{\mathrm{d}}{\mathrm{d} z} \log \Gamma_{\lambda}^{*}(z)=\frac{\Gamma_{\lambda}^{*}(z)}{\Gamma_{\lambda}^{*}(z)},
$$

where $\Gamma_{\lambda}^{*}(z)$ is the degenerate gamma function defined by (1). Now, we are going to obtain certain functional equations involving the degenerate digamma function $\psi_{\lambda}^{*}(z)$. Using (2) and (21), it follows that

$$
\begin{aligned}
\psi_{\lambda}^{*}(z+1) & =\frac{\Gamma_{\lambda}^{*^{\prime}}(z+1)}{\Gamma_{\lambda}^{*}(z+1)}=\frac{\left(z \Gamma_{\lambda}^{*}(z)\right)^{\prime}}{z \Gamma_{\lambda}^{*}(z)} \\
& =\frac{\Gamma_{\lambda}^{*^{\prime}}(z)}{\Gamma_{\lambda}^{*}(z)}+\frac{1}{z}=\psi_{\lambda}^{*}(z)+\frac{1}{z},
\end{aligned}
$$

$$
\operatorname{Re}(z)>0 \text {. }
$$

Generally, we have the following.

Theorem 3. For $n \in \mathbb{N}, z \in \mathbb{C}$, and $\operatorname{Re}(z)>0$, we have

$$
\psi_{\lambda}^{*}(z+n)=\psi_{\lambda}^{*}(z)+\sum_{m=0}^{n-1} \frac{1}{z+m} .
$$

Furthermore, using relation (5), we find that

$$
\psi_{\lambda}^{*}(z)=\psi(z)+\log \left(\frac{\lambda}{\log (1+\lambda)}\right)
$$

where $\psi$ is the digamma function defined by (20). According to Batir [28], we have

$$
\psi(z)=-\gamma+\sum_{n=0}^{\infty} \frac{z-1}{(n+1)(n+z)}
$$

where

$$
\gamma=\lim _{n \longrightarrow \infty}\left(\sum_{k=1}^{n} \frac{1}{k}-\log n\right)=-0.577215
$$

is the Euler-Mascheroni constant. Hence, substituting (25) into (24), one gets the following.

Theorem 4. For $z \in \mathbb{C}, \quad \operatorname{Re}(z)>0$, and $\lambda \in(0,1)$,

$$
\psi_{\lambda}^{*}(z)=\log \left(\frac{\lambda}{\log (1+\lambda)}\right)-\lim _{n \longrightarrow \infty}\left[\log n-\sum_{j=0}^{n} \frac{1}{z+j}\right],
$$

$$
\psi_{\lambda}^{*}(z)=\log \left(\frac{\lambda}{\log (1+\lambda)}\right)-\gamma+(z-1) \sum_{n=0}^{\infty} \frac{1}{(n+1)(n+z)}
$$

$\psi_{\lambda}^{*}(z+1)=\log \left(\frac{\lambda}{\log (1+\lambda)}\right)-\gamma+z \sum_{n=1}^{\infty} \frac{1}{n(n+z)}$

Next, the degenerate digamma function $\psi_{\lambda}^{*}(z)$ defined by (21) can be expressed as a series expression in terms of Riemann's zeta function. Using

$$
(n+z)^{-1}=n^{-1} \sum_{m=0}^{\infty}\left(\frac{-z}{n}\right)^{m}
$$

equation (29) can be rewritten as

$$
\psi_{\lambda}^{*}(z+1)=\log \left(\frac{\lambda}{\log (1+\lambda)}\right)-\gamma-\sum_{n=1}^{\infty} \sum_{m=1}^{\infty} n^{-(m+1)}(-z)^{m} .
$$

Thus, one gets the following.

Theorem 5. For $z \in \mathbb{C}, \operatorname{Re}(z)>0$, and $\lambda \in(0,1)$,

$$
\psi_{\lambda}^{*}(z+1)=\log \left(\frac{\lambda}{\log (1+\lambda)}\right)-\gamma-\sum_{m=1}^{\infty} \zeta(m+1)(-z)^{m} .
$$

Note that these series converge absolutely for $|z|<1$.

Using the Legendre duplication formula [29]

$$
\Gamma\left(\frac{1}{2}\right) \Gamma(2 z)=2^{2 z-1} \Gamma(z) \Gamma\left(z+\frac{1}{2}\right)
$$

and (5), one can simply find

$$
\Gamma_{\lambda}^{*}\left(\frac{1}{2}\right) \Gamma_{\lambda}^{*}(2 z)=2^{2 z-1} \Gamma_{\lambda}^{*}(z) \Gamma_{\lambda}^{*}\left(z+\frac{1}{2}\right),
$$

$$
\psi_{\lambda}^{*}(2 z)=\log 2+\frac{1}{2} \psi_{\lambda}^{*}(z)+\frac{1}{2} \psi_{\lambda}^{*}\left(z+\frac{1}{2}\right), \quad \operatorname{Re}(z)>0 .
$$

Equation (35) can be extended to an arbitrary integral multiplication of $z$ as follows. 
Theorem 6. For $z \in \mathbb{C}, \operatorname{Re}(z)>0$, and $\lambda \in(0,1)$,

$$
\psi_{\lambda}^{*}(m z)=\log m+\frac{1}{m} \sum_{j=1}^{m} \psi_{\lambda}^{*}\left(z+\frac{j-1}{m}\right), \quad \operatorname{Re}(z)>0 .
$$

Figures 1-3 illustrate the degenerate digamma function $\psi_{\lambda}^{*}(z)$ in $(24)$ at different values for $\lambda \in(0,1)$.

Remark 3. Its worth to mention here that all plotted functions in the below figures were multiplied by $\sin x$, since Fourier space, for the sake of clarify the results to the reader.

Now, we are going to find the integral representations for the degenerate digamma function $\psi_{\lambda}^{*}(z)$, defined by (21), as follows. Note that

$$
\begin{aligned}
\int_{0}^{1}\left(1-t^{z-1}\right)(1-t)^{-1} \mathrm{~d} t & =\sum_{n=0}^{\infty} \int_{0}^{1}\left(1-t^{z-1}\right) t^{n} \mathrm{~d} t \\
& =(z-1) \sum_{n=0}^{\infty} \frac{1}{(n+1)(n+z)}
\end{aligned}
$$

Hence, using (28) and (37), it can be shown that $\psi_{\lambda}^{*}(z)=-\gamma+\log \left(\frac{\lambda}{\log (1+\lambda)}\right)+\int_{0}^{1}\left(1-t^{z-1}\right)(1-t)^{-1} \mathrm{~d} t$.
Now, substituting $t=(1+\lambda)^{-s / \lambda}$ in (37) gives

$$
\begin{aligned}
\psi_{\lambda}^{*}(z)= & -\gamma+\log \left(\frac{\lambda}{\log (1+\lambda)}\right)+\frac{\log (1+\lambda)}{\lambda} \\
& \times \int_{0}^{\infty}\left[(1+\lambda)^{-t / \lambda}-(1+\lambda)^{-z t / \lambda}\right]\left[1-(1+\lambda)^{-t / \lambda}\right]^{-1} \mathrm{~d} t .
\end{aligned}
$$

Since

$$
z^{-1}=\frac{\log (1+\lambda)}{\lambda} \int_{0}^{\infty}(1+\lambda)^{-z t / \lambda} \mathrm{d} t
$$

and by integrating from 1 to $n$, it follows that

$$
\begin{aligned}
\log n & =\int_{0}^{\infty} \int_{1}^{n}(1+\lambda)^{-z t / \lambda} \cdot \log (1+\lambda)^{1 / \lambda} \mathrm{d} z \mathrm{~d} t \\
& =\int_{0}^{\infty} \int_{1}^{n} \frac{1}{t} \mathrm{~d}_{z}(1+\lambda)^{-z t / \lambda} \mathrm{d} t \\
& =\int_{0}^{\infty} \frac{1}{t}\left[(1+\lambda)^{-t / \lambda}-(1+\lambda)^{-n t / \lambda}\right] \mathrm{d} t .
\end{aligned}
$$

Inserting (41) and

$$
(z+j)^{-1}=\frac{\log (1+\lambda)}{\lambda} \int_{0}^{\infty}(1+\lambda)^{-(z+j) t / \lambda} \mathrm{d} t
$$

in (27), we get

$$
\begin{aligned}
\psi_{\lambda}^{*}(z)= & \log \left(\frac{\lambda}{\log (1+\lambda)}\right)+\lim _{n \longrightarrow \infty} \int_{0}^{\infty}\left[\left((1+\lambda)^{-t / \lambda}-(1+\lambda)^{-n t / \lambda}\right) \frac{1}{t}-\sum_{j=0}^{n} \frac{\log (1+\lambda)}{\lambda}(1+\lambda)^{-(z+j) t / \lambda}\right] \mathrm{d} t \\
= & \log \left(\frac{\lambda}{\log (1+\lambda)}\right)+\lim _{n \longrightarrow \infty} \int_{0}^{\infty}\left\{(1+\lambda)^{-\frac{t}{\lambda}} t^{-1}-\frac{\log (1+\lambda)}{\lambda}(1+\lambda)^{-\frac{z t}{\lambda}}\left[1-(1+\lambda)^{-\frac{t}{\lambda}}\right]^{-1}\right\} \mathrm{d} t \\
& -\lim _{n \longrightarrow \infty} \int_{0}^{\infty}(1+\lambda)^{-\frac{n t}{\lambda}}\left\{t^{-1}-\frac{\log (1+\lambda)}{\lambda}(1+\lambda)^{-\frac{z t}{\lambda}}\left[1-(1+\lambda)^{-\frac{t}{\lambda}}\right]^{-1}\right\} \mathrm{d} t .
\end{aligned}
$$

Since the last limit equals to zero, it follows

$$
\begin{aligned}
\psi_{\lambda}^{*}(z)= & \log \left(\frac{\lambda}{\log (1+\lambda)}\right)+\int_{0}^{\infty}\left[\frac{1}{t}(1+\lambda)^{-t / \lambda}\right. \\
& \left.-\frac{\log (1+\lambda)}{\lambda}\left(1-(1+\lambda)^{-t / \lambda}\right)^{-1}(1+\lambda)^{-z t / \lambda}\right] \mathrm{d} t
\end{aligned}
$$

The following theorem summarizes the above results.

Theorem 7. For $z \in \mathbb{C}, \operatorname{Re}(z)>0$, and $\lambda \in(0,1)$, the degenerate digamma function $\psi_{\lambda}^{*}(z)$, defined by (10), can be expressed as (38), (39) as well as (44).

\section{Degenerate Polygamma Function}

The polygamma function of order $m$ is obtained by taking the $(m+1)$ th derivative of the logarithm of gamma function (cf. [28]). Thus,

$$
\psi^{(m)}(z)=\frac{\mathrm{d}^{m}}{\mathrm{~d} z^{m}} \psi(z)=\frac{\mathrm{d}^{m+1}}{\mathrm{~d} z^{m+1}} \log \Gamma(z), \quad \operatorname{Re}(z)>0 .
$$

In this section, we define the degenerate polygamma function of order $m$ as

$$
\psi_{\lambda}^{*(m)}(z)=\frac{\mathrm{d}^{m}}{\mathrm{~d} z^{m}} \psi_{\lambda}^{*}(z)=\frac{\mathrm{d}^{m+1}}{\mathrm{~d} z^{m+1}} \log \Gamma_{\lambda}^{*}(z), \quad \operatorname{Re}(z)>0,
$$




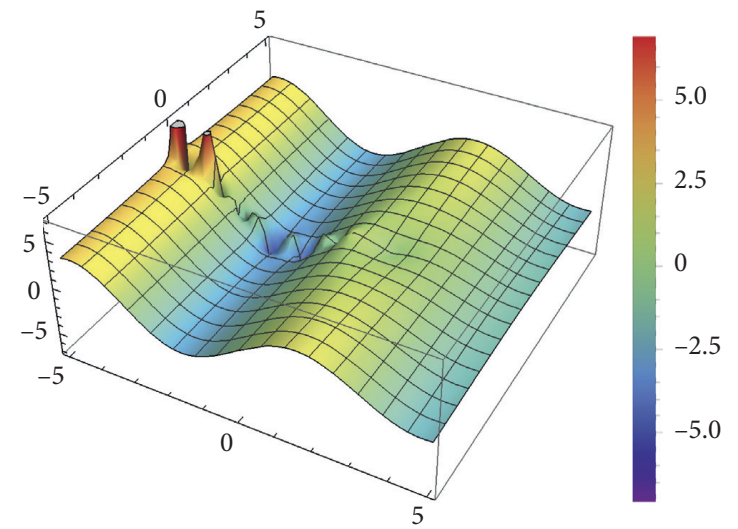

(a)

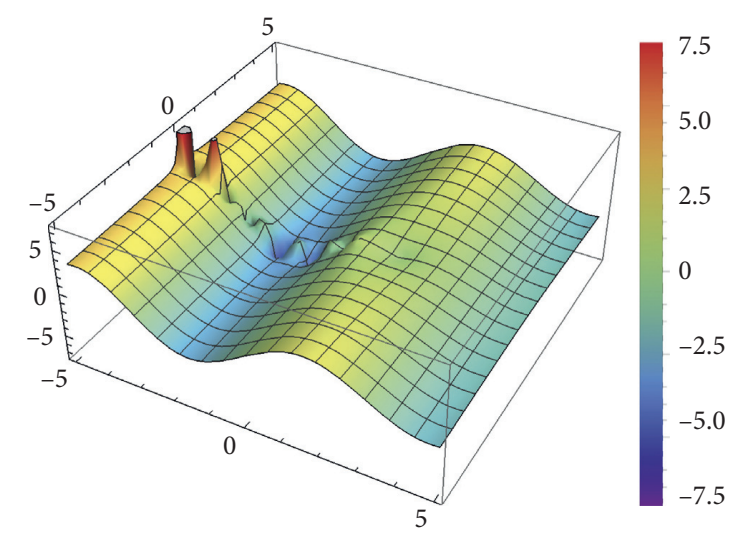

(b)

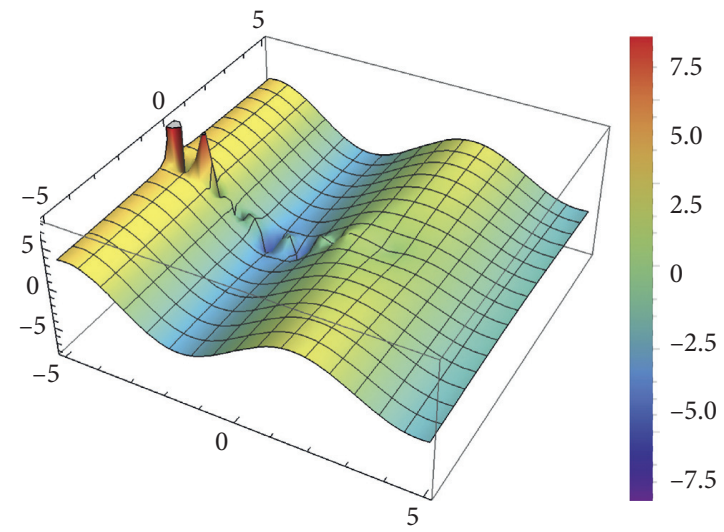

(c)

Figure 1: Absolute plots of the degenerate digamma function. (a) $\lambda=0.1$. (b) $\lambda=0.5$. (c) $\lambda=1.0$.

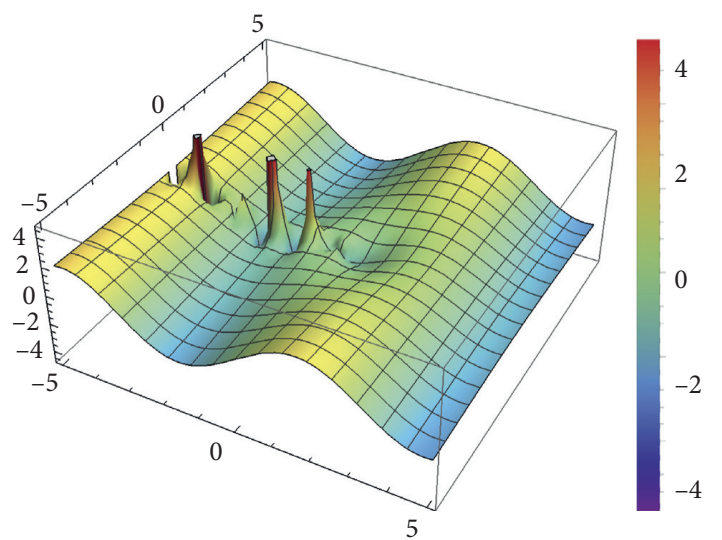

(a)

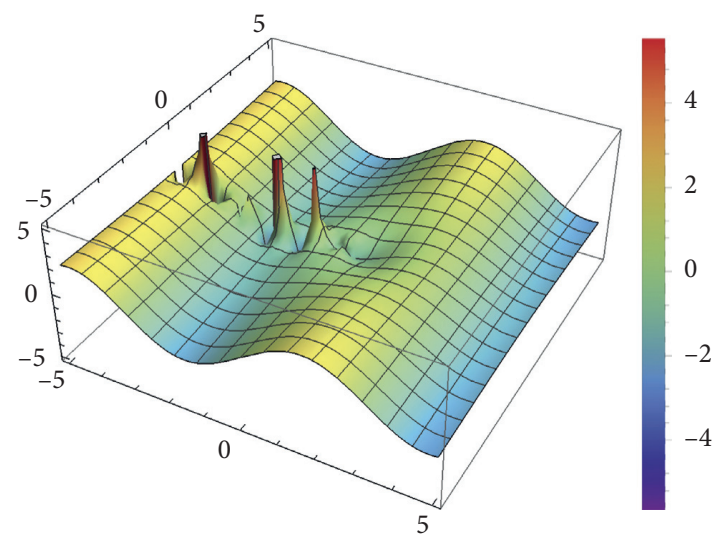

(b)

FIgure 2: Continued. 


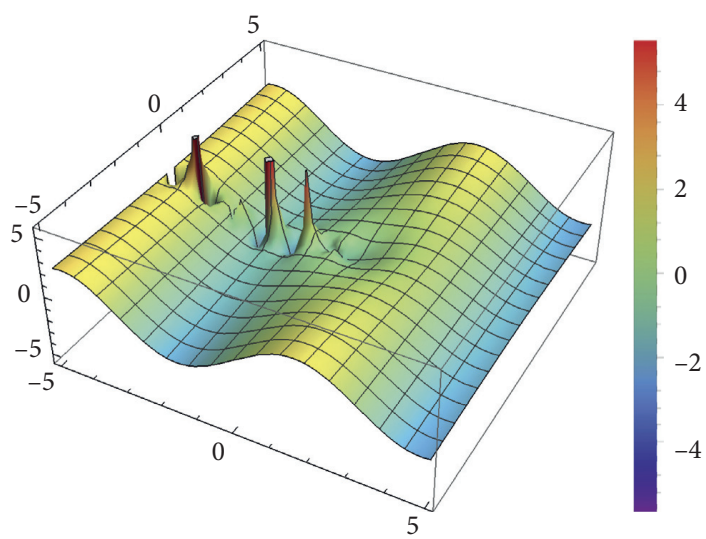

(c)

FIGURE 2: Real-part plots of the degenerate digamma function. (a) $\lambda=0.1$. (b) $\lambda=0.5$. (c) $\lambda=1.0$.

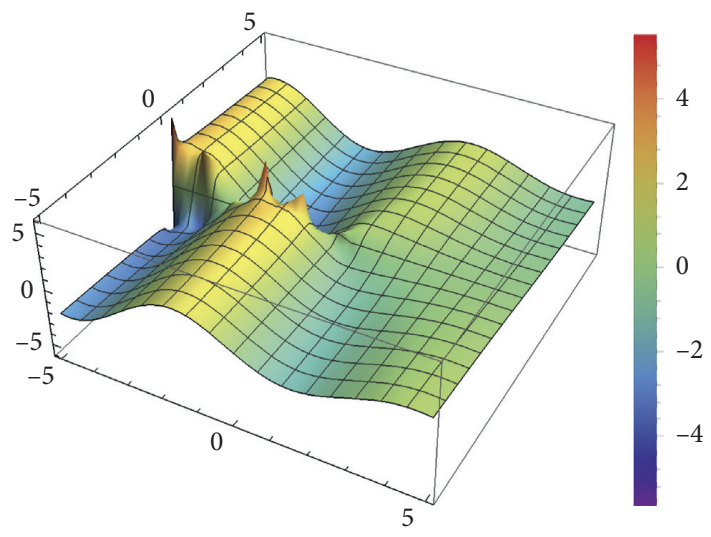

(a)

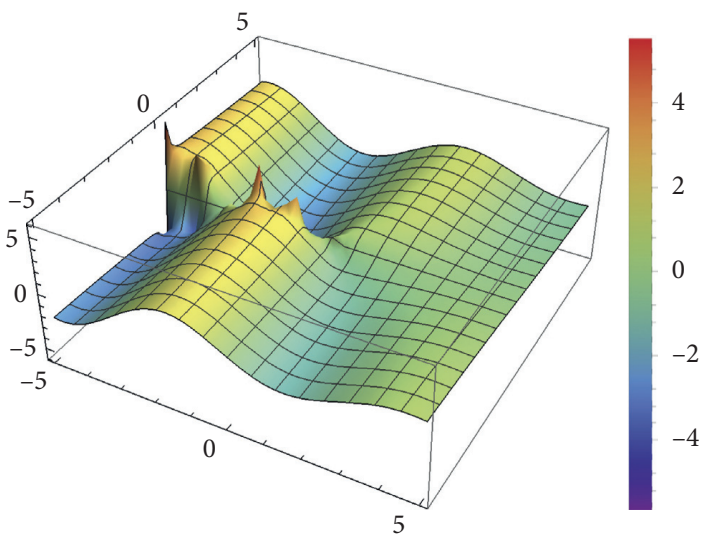

(b)

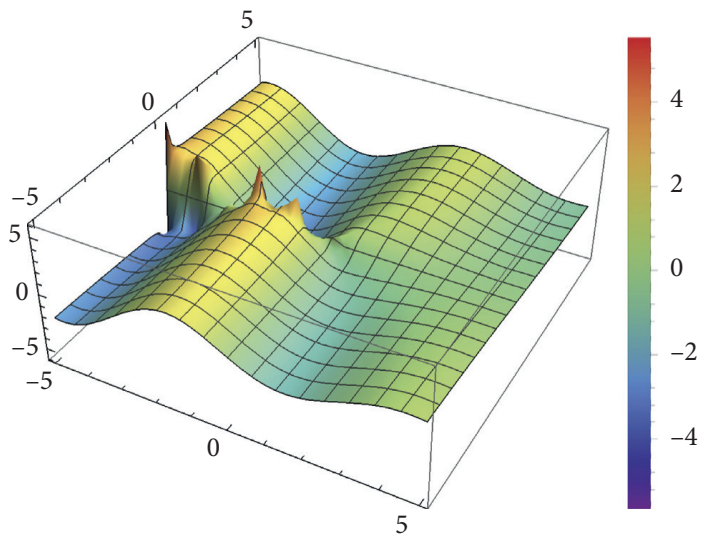

(c)

FIGURE 3: Imagery-part plots of the degenerate digamma function. (a) $\lambda=0.1$. (b) $\lambda=0.5$. (c) $\lambda=1.0$.

where $\Gamma_{\lambda}^{*}(z)$ is the degenerate gamma function defined by (1) and $\psi_{\lambda}^{*}(z)$ is the degenerate digamma function defined by (21).

By (24), it follows that

$$
\psi_{\lambda}^{*(m)}(z)=\psi^{(m)}(z), \quad \operatorname{Re}(z)>0 .
$$

Using (44), an integral representation for $\psi_{\lambda}^{*(m)}(z)$, given in the next theorem, can be obtained.

Theorem 8. Let $\lambda \in(0,1)$ and $m \in \mathbb{N}$. Then, for $z \in \mathbb{C}$ with $\operatorname{Re}(z)>0$, the degenerate polygamma function $\psi_{\lambda}^{*(m)}(z)$, defined by (46), can be expressed as 


$$
\begin{aligned}
\psi_{\lambda}^{*(m)}(z)= & (-1)^{m}\left(\frac{\log (1+\lambda)}{\lambda}\right)^{m+1} \\
& \times \int_{0}^{\infty} t^{m}\left[1-(1+\lambda)^{-t / \lambda}\right]^{-1}(1+\lambda)^{-z t / \lambda} \mathrm{d} t
\end{aligned}
$$

The following recurrence relations for the degenerate polygamma function $\psi_{\lambda}^{*(m)}(z)$ defined by (47) can be obtained from (22)-(24), (35), and (36) as the following.

Theorem 9. For $z \in \mathbb{C}, \operatorname{Re}(z)>0, \lambda \in(0,1)$, and $m \in \mathbb{N}$, the recurrence relations hold true:

$$
\begin{array}{r}
\psi_{\lambda}^{*(m)}(z+1)=\psi_{\lambda}^{*(m)}(z)+\frac{(-1)^{m} \Gamma(m+1)}{z^{m+1}}, \\
\psi_{\lambda}^{*(m)}(1-z)=(-1)^{m} \psi_{\lambda}^{*(m)}(z)+(-1)^{m} \pi\left(\frac{\mathrm{d}}{\mathrm{d} z}\right)^{m} \cot (\pi z), \\
\psi_{\lambda}^{*(m)}(z+n)=\psi_{\lambda}^{*(m)}(z)+\sum_{k=0}^{n-1} \frac{(-1)^{m} \Gamma(m+1)}{(z+k)^{m+1}}, \\
\psi_{\lambda}^{*(m)}(2 z)=\frac{1}{4} \psi_{\lambda}^{*(m)}(z)+\frac{1}{4} \psi_{\lambda}^{*(m)}\left(z+\frac{1}{2}\right), \\
\psi_{\lambda}^{*(m)}(n z)=\frac{1}{n^{m+1}} \sum_{k=1}^{n} \psi_{\lambda}^{*(m)}\left(z+\frac{k-1}{n}\right), \quad \operatorname{Re}(z)>0 .
\end{array}
$$

From (25), a series representation of the degenerate polygamma function $\psi_{\lambda}^{*(m)}(z)$ is given in the following result.

Theorem 10. For $z \in \mathbb{C}, \operatorname{Re}(z)>0, \lambda \in(0,1)$, and $m \in \mathbb{N}$, we have

$$
\psi_{\lambda}^{*(m)}(z)=(-1)^{m+1} \Gamma(m+1) \sum_{n=0}^{\infty} \frac{1}{(z+n)^{m+1}} .
$$

Remark 4. The degenerate polygamma function $\psi_{\lambda}^{*(m)}(z)$ can be expressed in terms of the generalized zeta function

$$
\zeta(m, z)=\sum_{n=0}^{\infty}(z+n)^{-m}
$$

as

$$
\psi_{\lambda}^{*(m)}(z)=(-1)^{m} \Gamma(m+1) \zeta(m+1, z) .
$$

Finally, using (32), a series representation in terms of the Riemann zeta function can be obtained, see the following result.

Theorem 11. For $z \in \mathbb{C}, \operatorname{Re}(z)>0, \lambda \in(0,1)$, and $m \in \mathbb{N}$, we have

$$
\psi_{\lambda}^{*(m)}(z+1)=\sum_{n=0}^{\infty}(-1)^{m+n+1} \Gamma(m+n+1) \zeta(m+n+1) \frac{z^{n}}{n !},
$$

$m, n \in \mathbb{N}$.

\section{Applications}

Let $z \in \mathbb{C}$ with $\operatorname{Re}(z)>0$ and $n \in \mathbb{N}$. Then, it can be verified that

$$
{ }_{3} F_{2}\left[\begin{array}{c}
(-n+2), z+1,1 \\
z+(n+1), 2
\end{array} ; 1\right]=\frac{z+n}{z(-n+1)} \times\left({ }_{2} F_{1}\left[\begin{array}{c}
(-n+1), z \\
z+1
\end{array} ; 1\right]-1\right) .
$$

Now, we can directly use the integral transform of Gauss hypergeometric function (see [29]) and the formulae:

$$
\begin{array}{r}
\Gamma\left(\frac{1}{2}\right) \Gamma(2 z)=2^{2 z-1} \Gamma(z) \Gamma\left(z+\frac{1}{2}\right), \quad \operatorname{Re}(z)>0, \\
2 F_{1}\left[\begin{array}{c}
(-n+2), z \\
z+1
\end{array} ;\right]=2^{-z} \frac{\Gamma(z+n) \Gamma(n-(1 / 2))}{\Gamma((z / 2)+n) \Gamma((z / 2)+(n-(1 / 2)))}, \\
\operatorname{Re}(z)>0 .
\end{array}
$$

Using (54) in (56) and L'Hôpital rule for complex numbers with applying equation (24) yields the following identity in terms of the degenerate digamma function:

$$
{ }_{3} F_{2}\left[\begin{array}{c}
(-n+2), z+1,1 \\
z+(n+1), 2
\end{array} ; 1\right]=\frac{z+1}{z} \times\left[\psi_{\lambda}^{*}\left(\frac{1}{2}\right)+\psi_{\lambda}^{*}(z+1)-\psi_{\lambda}^{*}\left(\frac{1}{2}(z+1)\right)-\psi_{\lambda}^{*}\left(\frac{1}{2} z+1\right)\right], \quad \operatorname{Re}(z)>0
$$


Similarly, we can present another identity involving hypergeometric function in terms of our degenerate digamma function in the following form:

$$
\begin{array}{r}
4 F_{3}\left[\begin{array}{c}
1,1,1,-n \\
2,2, z+1
\end{array} ; 1\right] \times\left[\left(\psi_{\lambda}^{*}(n+2)-\log \left(\frac{\lambda}{\log (1+\lambda)}\right)\right)\left(\psi_{\lambda}^{*}(z+n+1)-\psi_{\lambda}^{*}(z)\right)\right. \\
\left.-\sum_{s=1}^{n} \frac{\psi_{\lambda}^{*}(s+1)-\log (\lambda / \log (1+\lambda))}{z+1}\right], \quad \operatorname{Re}(\mathrm{z})>0 .
\end{array}
$$

\section{Data Availability}

Data sharing is not applicable to this article as no datasets were generated or analyzed during the current study.

\section{Conflicts of Interest}

The authors declare that they have no conflicts of interest.

\section{Authors' Contributions}

All the authors contributed equally and significantly to writing this article. All the authors read and approved the final manuscript.

\section{Acknowledgments}

The authors wish to acknowledge the approval and the support of this research study from the Deanship of Scientific Research in King Khalid University, Abha, Saudi Arabia, under grant R.G.P-79-41. The first and second authors wish to acknowledge the approval and the support of this research study from the National Natural Science Foundation of China (11601525). The authors are thankful to Professor Hillal Elshehabey for the figures illustrated in this paper.

\section{References}

[1] P. Agarwal, F. Qi, M. Chand, and G. Singh, "Some fractional differential equations involving generalized hypergeometric functions," Journal of Applied Analysis, vol. 25, no. 1, pp. 37-44, 2019.

[2] P. Agarwal, T. Rassias, G. Singh, and S. Jain, "Certain fractional integral and differential formulas involving the extended incomplete generalized hypergeometric functions," in Mathematical Analysis and Applications, pp. 217-272, Springer, Cham, Switzerland, 2019.

[3] M. Chand and Z. Hammouch, "Unified fractional integral formulae involving generalized multiindex Bessel function," in International Conference on Computational Mathematics and Engineering Sciences, pp. 278-290, Springer, Cham, Switzerland, 2019, https://doi.org/10.1007/978-3-030-391126_22.

[4] M. Chand, P. Agarwal, and Z. Hammouch, "Certain sequences involving product of k-Bessel function,"
International Journal of Applied and Computational Mathematics, vol. 4, no. 4, p. 101, 2018.

[5] C. A. Braumann, J.-C. Cortés, L. Jódar, and L. Villafuerte, “On the random gamma function: theory and computing," Journal of Computational and Applied Mathematics, vol. 335, pp. 142-155, 2018.

[6] L. Yin, L. Huang, L. Song, and X. K. Dou, "Some monotonicity properties and inequalities for the generalized digamma and polygamma functions," Journal of Inequalities and Applications, vol. 2018, Article ID 249, 2018.

[7] J. Jiang and L. Liu, "Existence of solutions for a sequential fractional differential system with coupled boundary conditions," Boundary Value Problems, vol. 2016, Article ID 159, 2016.

[8] X. Lin and Z. Zhao, "Iterative technique for a third-order differential equation with three-point nonlinear boundary value conditions," Electronic Journal of Qualitative Theory of Differential Equations, vol. 2016, no. 12, pp. 1-10, 2016.

[9] R. Xu and F. Meng, "Some new weakly singular integral inequalities and their applications to fractional differential equations," Journal of Inequalities and Applications, vol. 2016, Article ID 78, 2016.

[10] H. Srivastava and J. Choi, Zeta and Q-Zeta Functions and Associated Series and Integrals, Elsevier, Inc., Amsterdam, Netherlands, 2012.

[11] J. Min, "Zeros and special values of Witten zeta functions and Witten L-functions," Journal of Number Theory, vol. 134, pp. 240-257, 2014.

[12] M. Kim, "Some series involving the Euler zeta function," Turkish Journal of Mathematics, vol. 42, no. 3, pp. 1166-1179, 2018.

[13] T. Kim, D. V. Dolgy, L.-C. Jang, and H.-I. Kwon, "Some identities of degenerate $\mathrm{q}$-Euler polynomials under the symmetry group of degree n," Journal of Nonlinear Sciences and Applications, vol. 9, no. 6, pp. 4707-4712, 2016.

[14] T. Kim, "Euler numbers and polynomials associated with Zeta functions," Abstract and Applied Analysis, vol. 2008, Article ID 581582, 11 pages, 2008.

[15] D. S. Kim, H. Y. Kim, D. Kim, and T. Kim, "Identities of symmetry for type 2 Bernoulli and euler polynomials," Symmetry, vol. 11, no. 5, p. 613, 2019.

[16] R. Dwivedi and V. Sahai, "On certain properties and expansions of zeta matrix function, digamma matrix function and polygamma matrix function," Quaestiones Mathematicae, vol. 43, no. 1, pp. 97-105, 2020.

[17] A. Bakhet, Y. Jiao, and F. He, "On the Wright hypergeometric matrix functions and their fractional calculus," Integral 
Transforms and Special Functions, vol. 30, no. 2, pp. 138-156, 2019.

[18] F. He, A. Bakhet, M. Hidan, and M. Abdalla, "Two variables shivley's matrix polynomials," Symmetry, vol. 11, no. 2, p. 151, 2019.

[19] M. Akel, A. Bakhet, and M. Abdalla, "On the matrix version of degenerate hypergeometric function," Linear Multilinear Algebra, 2020, In press.

[20] He. Fuli, A. Bakhet, M. Hidan, and M. Abdalla, "On the extended hypergeometric matrix functions and their applications for the derivatives of the extended Jacobi matrix polynomial," Mathematical Problems in Engineering, vol. 2020, Article ID 4268361, 8 pages, 2020.

[21] A. Bakhet and F. He, "On 2-variables Konhauser matrix polynomials and their fractional integrals," Mathematics, vol. 8 , no. 2, p. 232, 2020.

[22] M. Hidan and M. Abdalla, "A note on the Appell hypergeometric matrix function," Mathematical Problems in Engineering, vol. 2020, Article ID 6058987, 6 pages, 2020.

[23] M. Abdalla, "Special matrix functions: characteristics, achievements and future directions," Linear and Multilinear Algebra, vol. 68, no. 1, pp. 1-28, 2020.

[24] T. Kim, "Degenerate Euler zeta function," Russian Journal of Mathematical Physics, vol. 22, no. 4, pp. 469-472, 2015.

[25] T. Kim and D. S. Kim, "Degenerate Laplace transform and degenerate gamma function," Russian Journal of Mathematical Physics, vol. 24, no. 2, pp. 241-248, 2017.

[26] Y. Kim, B. Kim, L. Jang, and J. Kwon, "A note on modified degenerate gamma and Laplace transformation," Symmetry, vol. 10, p. 471, 2019.

[27] T. Kim and G. Jang, "A note on degenerate gamma function and degenerate Stirling number of the second kind," Advanced Studies in Contemporary Mathematics, vol. 28, pp. 207-214, 2018.

[28] N. Batir, "On some properties of digamma and polygamma Functions," Journal of Mathematical Analysis and Applications, vol. 328, no. 1, pp. 452-465, 2007.

[29] L. Debnath and D. Bhatta, Integral Transform and Their Application, CRC Press, Boca Raton, FL, USA, 2015. 